\title{
Influência da adição de níquel na síntese do $\mathrm{SrSnO}_{3}$
}

\section{(Influence of nickel doping on the $\mathrm{SrSnO}_{3}$ synthesis)}

\author{
M. R. Nascimento ${ }^{1,2}$, M. R. C. Santos ${ }^{3}$, S. J. G. Lima ${ }^{4}$, C. D. Pinheiro ${ }^{5}$, J. W. M. Espinosa ${ }^{6}$, E. Longo , $^{6}$ \\ A. G. Souza ${ }^{1}$, I. M. G. Santos ${ }^{1}$ \\ ${ }^{1}$ Departamento de Química, CCEN, Universidade Federal da Paraíba, J. Pessoa, PB 58059-900 \\ ${ }^{2}$ Escola Agrotécnica Federal de Sousa - EAFS-PB \\ ${ }^{3}$ Instituto de Química, Universidade Federal da Goiás, Catalão, GO \\ ${ }^{4}$ Departamento de Engenharia Mecânica, CT, Universidade Federal da Paraíba, J. Pessoa, PB \\ ${ }^{5} U A C E N, C F P$, Universidade Federal de Campina Grande, Cajazeiras, PB \\ ${ }^{6} C M D M C / L I E C$, Instituto de Química, UNESP, Araraquara, SP \\ marceloquimica@gmail.com
}

\begin{abstract}
Resumo
Pós cerâmicos microestruturados de $\mathrm{Sr}_{1-\mathrm{x}} \mathrm{Ni}_{\mathrm{x}} \mathrm{SnO}_{3}$ (x de 0 a 0,20 ) foram sintetizados com sucesso pelo método dos precursores poliméricos. Os precursores foram caracterizados por análise térmica. Três etapas de decomposição foram observadas: a primeira relacionada com o processo de desidratação, a segunda e a terceira são processos de combustão da matéria orgânica. A caracterização estrutural foi feita por difração de raios X, espectrofotometria de absorção óptica no infravermelho e espectroscopia Raman após calcinação a $700{ }^{\circ} \mathrm{C}$, indicando a presença da fase $\mathrm{SrSnO}_{3}$ ortorrômbica. Quando níquel II foi adicionado na rede cristalina do estanato, foi observada uma transição de fase, conduzindo a uma nova estrutura pseudo-cúbica. Com base nos resultados de Raman e difração de raios X, foi analisada a ordem-desordem a curto e longo alcance. Os resultados experimentais mostram a formação de fases secundárias quando níquel II é adicionado na matriz, com calcinação a $700{ }^{\circ} \mathrm{C}$.
\end{abstract}

Palavras-chave: $\mathrm{SrSnO}_{3}$, perovskita, Pechini.

\begin{abstract}
$\mathrm{Sr}_{1-x} \mathrm{Ni}_{x} \mathrm{SnO}_{3}$ (x from 0 to 0.20) powders were synthesized by the polymeric precursor method. The powder precursors were characterized by thermal analysis. Three decomposition steps were observed: the first step related to the dehydration process, the second and third ones assigned to combustion processes. The structural characterization was done by X-ray diffraction (XRD), infrared absorption spectroscopy and Raman spectroscopy after calcination at $700{ }^{\circ} \mathrm{C}$, indicating the presence of orthorhombic $\mathrm{SrSnO}_{3}$. When nickel is added to the structure, a phase transition is observed, leading to the presence of a pseudo-cubic phase. Based on Raman and XRD results, the order-disorder at short and long range were analyzed. The experimental results show the formation of a secondary phase when nickel is added to the material and calcined at $700^{\circ} \mathrm{C}$.
\end{abstract}

Keywords: $\mathrm{SrSnO}$, perovskite, Pechini.

\section{INTRODUÇÃO}

Os estanatos de metais alcalinos terrosos, que têm a fórmula geral $\mathrm{MSnO}_{3}$ e $\mathrm{M}_{2} \mathrm{SnO}_{4}(\mathrm{M}=\mathrm{Ca}, \mathrm{Sr}$ e $\mathrm{Ba})$ têm sido estudados, recentemente, por apresentar propriedades muito interessantes, com grande potencial para aplicação em eletrônica, como em capacitores termicamente estáveis com baixa permissividade, além de sensores de vários gases, incluindo $\mathrm{CO}, \mathrm{NO}_{\mathrm{x}}$ e $\mathrm{H}_{2} \mathrm{O}$, e catalisadores, visando reduzir a quantidade produzida de substâncias poluentes, tal como $\mathrm{NO}_{\mathrm{x}}$ [1-17]. Além disso, os sistemas à base de níquel ou dopados com níquel são conhecidos como excelentes catalisadores para diferentes substâncias gasosas orgânicas e inorgânicas, como hidrocarbonetos, óxidos de nitrogênio $\left(\mathrm{NO}_{\mathrm{x}}\right)$ e monóxido de carbono [18-22 ].

Diversos trabalhos já foram publicados, descrevendo a síntese de estanatos: reação do estado sólido [23], coprecipitação e combustão [24], síntese hidrotermal [25], precursores peróxidos [26] e polimerização pelo método complexo polimerizado (PC) [12], que é mais uma variante do método Pechini $[27,28]$. Um dos métodos de síntese que vem se popularizando na produção de pós e filmes cerâmicos [29-31] é o método dos precursores poliméricos, derivado do método Pechini, que se baseia na obtenção de poliésteres a partir de citratos. As vantagens desse método, quando comparado aos tradicionais, são homogeneidade química dos multicomponentes em escala atômica, controle direto e preciso da estequiometria de sistemas complexos em temperaturas relativamente baixas, pós-cerâmicos com partículas muito finas e simplicidade de processamento e flexibilidade, podendo ser empregado na obtenção de diversos óxidos, com diferentes estruturas por simples ajuste de variáveis. 
$\mathrm{O}$ estanato do estrôncio, $\mathrm{SrSnO}_{3}$, a mais estudada destas perovskitas, é um material dielétrico de importância tecnológica, sendo sintetizado normalmente em temperaturas acima de $1000{ }^{\circ} \mathrm{C}$ por reação no estado sólido entre $\mathrm{SrCO}_{3}$ ou $\mathrm{SrO}$ e $\mathrm{SnO}_{2}$, cristalizando-se no sistema pseudo-cúbico, com estrutura perovskita. Como altas temperaturas de preparação são normalmente utilizadas, são formados, freqüentemente, pós de tamanho de grão relativamente grandes e com certo grau de impureza. Existem poucos trabalhos sobre a síntese desse material por via úmida.

Alguns autores consideram o $\mathrm{SrSnO}_{3}$ como uma perovskita cúbica, após calcinação acima de $1050{ }^{\circ} \mathrm{C}$, mas há relatos de que $\mathrm{SrSnO}_{3}$ tem algum desvio da estrutura cúbica ideal. Não há muita similaridade entre os espectros Raman desse material com o da perovskita cúbica pura, que tem picos largos característicos [29].

O objetivo desse trabalho consiste em sintetizar $\mathrm{Sr}_{1-\mathrm{x}} \mathrm{Ni}_{\mathrm{x}} \mathrm{SnO}_{3}$ (x de 0 a 0,2), utilizando o método dos precursores poliméricos. A dopagem com $\mathrm{Ni}^{2+}$ tem como intenção gerar defeitos estruturais na rede cristalina receptora e inserir orbitais $d$ nessa estrutura, de modo a ampliar as aplicações tecnológicas desse material.

\section{EXPERIMENTAL}

Os reagentes usados na síntese da perovskita estão listados na Tabela I. Citrato de estanho foi usado para a preparação das resinas precursoras dos pós de $\mathrm{Sr}_{1-\mathrm{x}} \mathrm{Ni}_{\mathrm{x}} \mathrm{SnO}_{3}(\mathrm{x}=0,0,01$, $0,05,0,1,0,15$ e 0,2$)$, tendo sido obtido a partir do cloreto de estanho [29]. Após a síntese desse citrato, os sais $\mathrm{Sr}\left(\mathrm{NO}_{3}\right)_{2}$ e $\mathrm{Ni}\left(\mathrm{CH}_{3} \mathrm{COO}\right)_{2} \cdot 4 \mathrm{H}_{2} \mathrm{O}$ foram adicionados lentamente na solução do citrato, até ocorrer o processo completo de dissolução. Foi utilizada uma relação molar 3:1 de ácido cítrico:metal para garantir a completa quelação dos metais na solução. Etileno glicol foi adicionado nessa solução em uma relação mássica de 60:40 de ácido cítrico:etileno glicol. A temperatura da reação foi controlada em $80{ }^{\circ} \mathrm{C}$, ocorrendo então o processo de polimerização. A resina foi aquecida a $300{ }^{\circ} \mathrm{C}$ ao ar durante $2 \mathrm{~h}$ para se obter o pó precursor. Esse pó foi desaglomerado passando por uma peneira de 200 mesh. A caracterização térmica dos precursores foi feita por termogravimetria (TG) e análise térmica diferencial (ATD).

As curvas TG/ATD foram obtidas em uma termobalança SDT 2960, TA Instruments, em atmosfera de $100 \mathrm{~mL}$. min ${ }^{1}$ de ar sintético. Nesta análise foram utilizados cadinhos de alumina, razão de aquecimento $10{ }^{\circ} \mathrm{C} / \mathrm{min}$, massa da amostra $10,0 \pm 0,5 \mathrm{mg}$ e em uma faixa de temperatura de 25 a $1200^{\circ} \mathrm{C}$.

Os pós precursores foram calcinados em atmosfera de ar sintético a $400{ }^{\circ} \mathrm{C}$ por $4 \mathrm{~h}$, seguido do tratamento térmico secundário a $700{ }^{\circ} \mathrm{C}$ por $4 \mathrm{~h}$, com uma taxa de aquecimento de $5{ }^{\circ} \mathrm{C} \cdot \mathrm{min}^{-1}$.

A caracterização da estrutura cristalina dos pós cerâmicos foi feita por difração de raios $\mathrm{X}$ em um difratômetro Siemens D-5000 na faixa de $2 \theta 10-90^{\circ}$ com passo $0,03^{\circ}$ e tempo por passo $1,0 \mathrm{~s}$, com radiação $\mathrm{K} \alpha$ do cobre à temperatura ambiente. Com os dados de DRX dos picos ( $\left.\begin{array}{lll}2 & 0 & 0\end{array}\right),\left(\begin{array}{lll}2 & 2 & 0\end{array}\right)$ e $\left(\begin{array}{lll}3 & 1 & 2\end{array}\right)$ foram calculados os parâmetros de rede com o programa Rede 93 [32] e a desordem a longo alcance, por meio da largura a meia altura do pico $(200)$.

Os espectros de absorção na região do infravermelho foram obtidos em amostras prensadas sob vácuo com $\mathrm{KBr}$ em um espectrofotômetro Bomem modelo MB 102. Os espectros foram registrados na região $4000-400 \mathrm{~cm}^{-1}$. As análises de espectroscopia Raman foram realizadas em um espectrofotômetro FT-Raman Bruker modelo RFS/100/ $\mathrm{S}$ usando um laser de Nd: YAG, com potência de $60 \mathrm{~mW}$ e comprimento de onda $1064 \mathrm{~nm}$, com resolução $4 \mathrm{~cm}^{-1}$, na região $10-1000 \mathrm{~cm}^{-1}$. Para análise da morfologia das partículas, após as calcinações, foi utilizado um microscópico eletrônico de varredura ZEISS DSM modelo $940 \mathrm{~A}$.

\section{RESULTADOS E DISCUSSÃO}

Os resultados da análise térmica dos pós precursores são apresentados na Fig. 1.

Em todas as curvas termogravimétricas analisadas são observadas quatro etapas de decomposição térmica. A primeira é atribuída à perda de $\mathrm{H}_{2} \mathrm{O}$ e gases adsorvidos na superfície do pó [33]. A segunda, a terceira e a quarta etapa

Tabela I - Propriedades dos reagentes.

[Table I - Properties of the reagents]

\begin{tabular}{lcccc}
\hline \multicolumn{1}{c}{ Reagentes } & $\begin{array}{c}\text { Fórmula } \\
\text { química }\end{array}$ & $\begin{array}{c}\mathrm{MM} \\
\left(\mathrm{g} \cdot \mathrm{mol}^{-1}\right)\end{array}$ & $\begin{array}{c}\text { Pureza } \\
(\%)\end{array}$ & Fornecedor \\
\hline Ácido cítrico & $\mathrm{C}_{6} \mathrm{H}_{8} \mathrm{O}_{7} \cdot \mathrm{H}_{2} \mathrm{O}$ & 192,13 & 99,5 & Cargill \\
Etileno glicol & $\mathrm{HO}_{\mathrm{CH}_{2}} \cdot \mathrm{CH}_{2} \cdot \mathrm{OH}$ & 62,07 & 99,0 & Vetec \\
Acetato de níquel & ${\mathrm{Ni}\left(\mathrm{CH}_{3} \mathrm{COO}_{2} \cdot 4 \mathrm{H}_{2} \mathrm{O}\right.}_{248,84}$ & 98,0 & Avocado \\
Nitrato de estrôncio & $\mathrm{Sr}^{\left(\mathrm{NO}_{3}\right)_{2}}$ & 211,63 & 99,0 & Vetec \\
Ácido nítrico & $\mathrm{HNO}_{3}$ & 62,997 & 65,0 & Dinâmica \\
Cloreto de estanho & $\mathrm{SnCl}_{2} \cdot 2 \mathrm{H}_{2} \mathrm{O}$ & 225,65 & 99,9 & Aldrich \\
Hidróxido de amônio & $\mathrm{NH}_{4} \mathrm{OH}$ & 35,04 & 99,0 & Nuclear \\
\hline
\end{tabular}



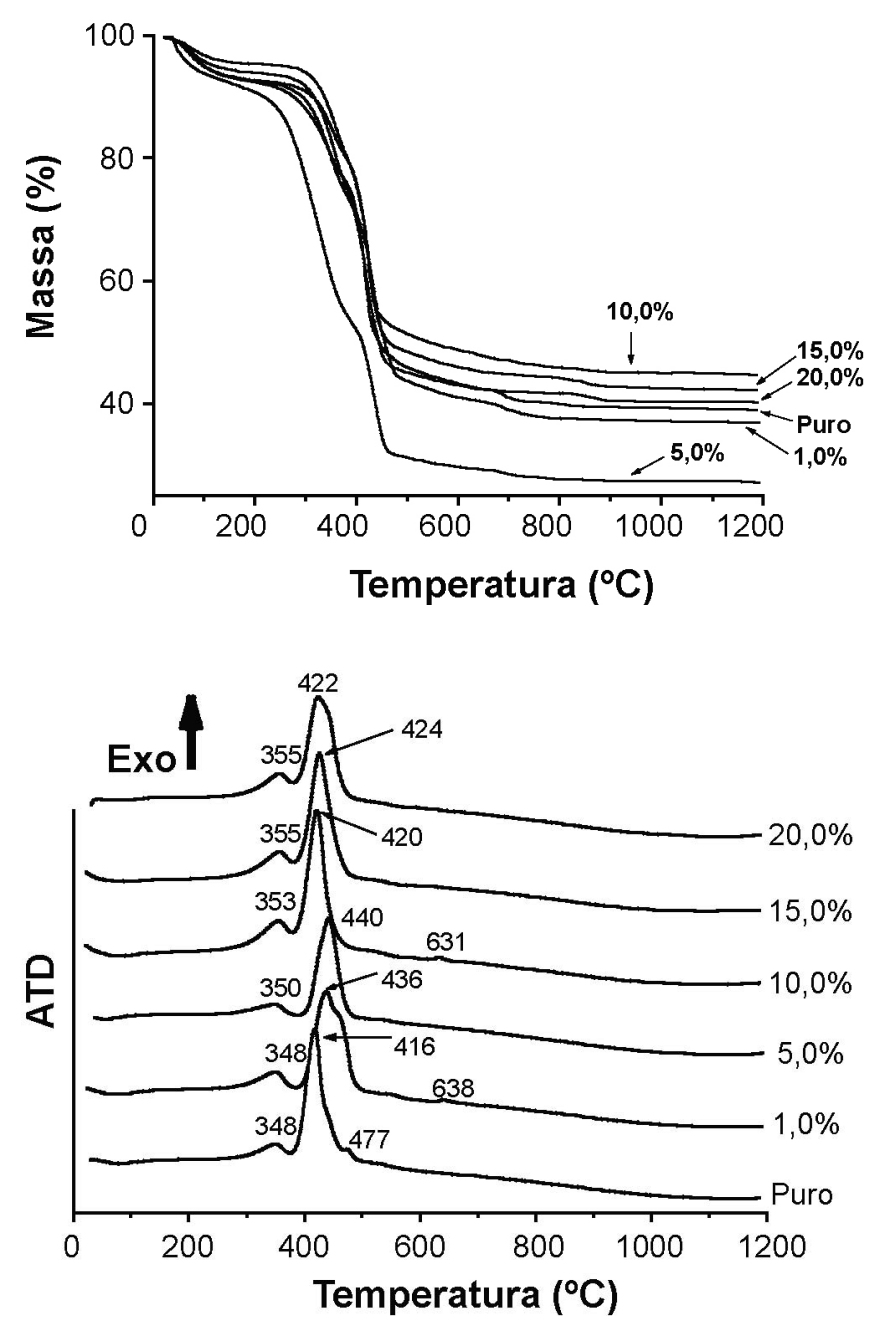

Figura 1: Curvas de análise térmica dos precursores $\mathrm{Sr}_{\mathrm{x}} \mathrm{Ni}_{1-\mathrm{x}} \mathrm{SnO}_{3}$. a: TG; b: ATD.

[Figure 1: Thermal analysis curves of the $\mathrm{Sr}_{x} \mathrm{Ni}_{1-x} \mathrm{SnO}_{3}$ powder precursors. $a$ : TG; $b$ : DTA.] estão relacionadas com a decomposição da matéria orgânica. Observa-se ainda que os pós de metaestanato de estrôncio puro perdem $51 \%$ da matéria orgânica por volta dos $690{ }^{\circ} \mathrm{C}$. Esses resultados são apresentados na Tabela II.

As curvas ATD (Fig. 1b) do precursor indicam picos exotérmicos. Estes picos sobrepõem com a segunda etapa de decomposição das curvas de TG, indicando a combustão do material orgânico. Dois picos foram observados, um menor a aproximadamente $350{ }^{\circ} \mathrm{C}$ e o mais intenso a $440{ }^{\circ} \mathrm{C}$. As amostras com 1 e $10 \%$ de níquel apresentam também um pequeno pico exotérmico a aproximadamente $635^{\circ} \mathrm{C}$, indicando a presença de compostos orgânicos refratários. Geralmente, uma baixa temperatura de decomposição é associada com um tamanho de partícula menor. A presença de picos largos indica a existência de uma larga distribuição no tamanho de partícula, em que a ruptura da cadeia polimérica pode estar ocorrendo de maneiras diferentes e por meio de várias etapas. Segundo os estudos anteriores [29], as curvas de TGATD para os precursores do $\mathrm{SrSnO}_{3}$ (derivados do método PC) apresentam um pico exotérmico em torno de $441{ }^{\circ} \mathrm{C}$, após combustão a $350^{\circ} \mathrm{C} / 2 \mathrm{~h}$

A Fig. 2 mostra os espectros de transmitância na região do infravermelho para as amostras $\mathrm{Sr}_{1-\mathrm{x}} \mathrm{Ni}_{\mathrm{x}} \mathrm{SnO}_{3}$ calcinadas a $700{ }^{\circ} \mathrm{C}$. Para a perovskita cúbica os modos normais de vibração da rede são dados na representação irredutível:

$$
\Gamma^{\text {total }}=4 \mathrm{~F}_{1 \mathrm{u}}+\mathrm{F}_{2 \mathrm{u}}
$$

Um dos modos $\mathrm{F}_{1 \mathrm{u}}$ corresponde ao modo acústico (fônon) e o modo $F_{2 u}$ é ótico, isto é, não é ativo em infravermelho ou Raman. Os três modos restantes $F_{i}$ são ativos no infravermelho, correspondendo, respectivamente, aos seguintes movimentos vibracionais: distância modular da ligação Sn-O (modo de estiramento), movimento translacional dos átomos de estrôncio na rede do $\mathrm{SnO}_{3}$ e do ângulo $\mathrm{Sn}-\mathrm{O}$ [34]. As vibrações do grupo do estanato $\left(\mathrm{SnO}_{3}^{2-}\right)$ são observadas em bandas de intensidade elevada, na faixa entre $300-400 \mathrm{~cm}^{-1}$

Tabela II - Temperatura e perda de massa dos pós precursores de $\mathrm{Sr}_{1-\mathrm{x}} \mathrm{Ni}_{\mathrm{x}} \mathrm{SnO}_{3}$ baseados nas curvas de TG e ATD. [Table II - Temperature and mass loss of precursor powders of $\mathrm{Sr}_{1-x} \mathrm{Ni}_{x} \mathrm{SnO}_{3}$ according to TG and DTA curves.]

Evento 2
Evento 3
Evento 4

\begin{tabular}{cccccccc}
$\begin{array}{c}\text { Amostra } \\
(\mathrm{mol} \% \mathrm{Ni})\end{array}$ & $\begin{array}{c}\text { Temperatura } \\
\left({ }^{\circ} \mathrm{C}\right)\end{array}$ & $\begin{array}{c}\text { Perda de } \\
\text { Massa }(\%)\end{array}$ & $\begin{array}{c}\text { Temperatura } \\
\left({ }^{\circ} \mathrm{C}\right)\end{array}$ & $\begin{array}{c}\text { Perda de } \\
\text { massa }(\%)\end{array}$ & $\begin{array}{c}\text { Temperatura } \\
\left({ }^{\circ} \mathrm{C}\right)\end{array}$ & $\begin{array}{c}\text { Perda de } \\
\text { massa }(\%)\end{array}$ & $\begin{array}{c}\text { ATD Temperatura } \\
\text { do pico }\left({ }^{\circ} \mathrm{C}\right)\end{array}$ \\
\hline 0 & $221-348$ & 11,39 & $348-416$ & 19,37 & $416-720$ & 20,81 & 348,416 e 477 \\
1,0 & $233-348$ & 11,66 & $348-436$ & 22,67 & $436-772$ & 19,38 & 348,436 e 638 \\
5,0 & $200-350$ & 23,52 & $350-440$ & 27,13 & $440-750$ & 11,91 & 350 e 440 \\
10,0 & $243-353$ & 8,38 & $353-420$ & 19,17 & $420-900$ & 19,56 & 353,420 e 631 \\
15,0 & $243-355$ & 11,39 & $355-424$ & 22,44 & $424-855$ & 18,04 & 355 e 424 \\
20,0 & $241-355$ & 13,42 & $355-422$ & 19,67 & $422-886$ & 19,96 & 355 e 422 \\
\hline
\end{tabular}




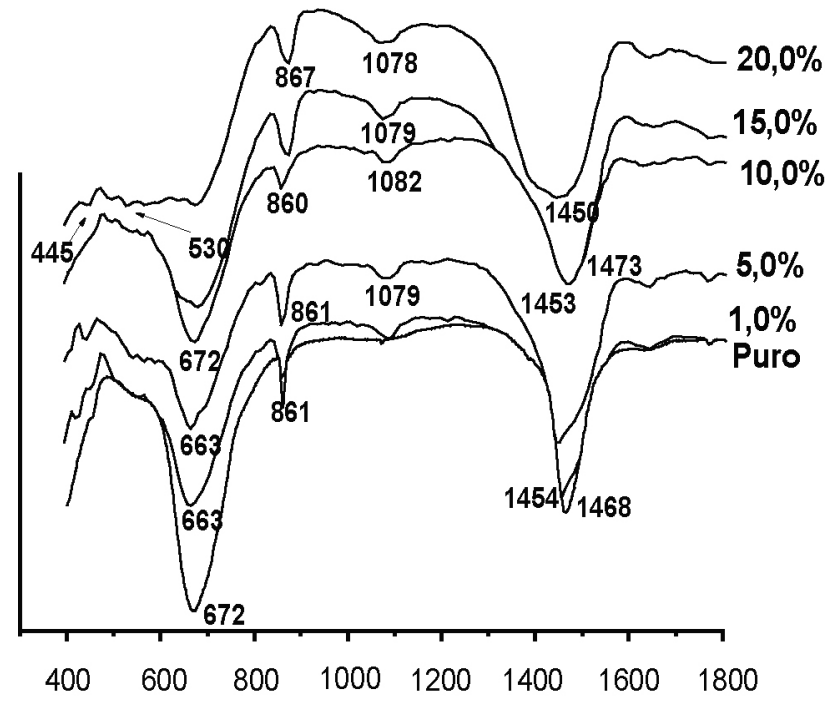

Número de onda $\left(\mathrm{cm}^{-1}\right)$

Figura 2: Espectros de absorção na região do infravermelho para $\mathrm{Sr}_{1-\mathrm{x}} \mathrm{Ni}_{\mathrm{x}} \mathrm{SnO}_{3}(\mathrm{x}=0,0,01,0,05,0,10,0,15$ e 0,20$)$ calcinados a $700{ }^{\circ} \mathrm{C}$.

[Figure 2: Infrared spectra of $\mathrm{Sr}_{1-x} \mathrm{Ni}_{x} \mathrm{SnO}_{3}$ powders $(x=0,0.01$, $0.05,0.10,0.15$ and 0.20 ), calcined at $700^{\circ} \mathrm{C}$.]

e em $600-700 \mathrm{~cm}^{-1}$ [35]. Nesse trabalho, verifica-se que esta última banda é observada em $669 \mathrm{~cm}^{-1}$. O aumento da concentração do dopante faz a banda em aproximadamente $668 \mathrm{~cm}^{-1}$ diminuir gradativamente. Com relação às bandas referentes às vibrações do grupo estanato $\left(\mathrm{SnO}_{3}^{2-}\right)$ e ao estiramento da ligação do Sn-O, há apenas um deslocamento em conseqüência da adição do dopante. A banda de absorção em torno de $450 \mathrm{~cm}^{-1}$ é atribuída ao estiramento da ligação Ni-O. Observa-se um aumento no número de bandas de pequena intensidade na região $460-560 \mathrm{~cm}^{-1}$. Esse fato está relacionado à maior dificuldade de organização do sistema, quando o $\mathrm{Ni}^{2+}$ é adicionado à rede. Esse fato advém da maior covalência do níquel, levando a uma ligação mais direcional e, conseqüentemente, mais distorcida. Como as bandas no infravermelho estão relacionadas à organização a curto alcance, elas se tornam menos definidas, especialmente a baixas temperaturas. De acordo com outros trabalhos anteriores, as duas bandas principais, características do carbonato do estrôncio observadas nos estanatos, são em 870 e $1430 \mathrm{~cm}^{-1}$ [34]. As bandas de absorção dos grupos carbonato são observadas em 1320-1530 $\mathrm{cm}^{-1}$ (forte), em 1040-1100 $\mathrm{cm}^{-1}$ (fraco) e em 800-890 $\mathrm{cm}^{-1}$ (médio) [35]. No presente trabalho, essas bandas são observadas a aproximadamente 1450,1080 e $860 \mathrm{~cm}^{-1}$. As intensidades dessas bandas decrescem com o aumento da concentração do dopante, mas não é uma redução significativa.

Os difratogramas de raios $\mathrm{X}$ das amostras de $\mathrm{Sr}_{1-\mathrm{X}} \mathrm{Ni}_{x} \mathrm{SnO}_{3}$ (x de 0 a 0,2 ), calcinados a $700{ }^{\circ} \mathrm{C}$ são apresentados na Fig. 3 e exibem os mesmos picos de difração, quando comparados à amostra não-dopada. É observada a formação de fases secundárias, de cassiterita $\left(\mathrm{SnO}_{2}\right.$ tetragonal $)$ e carbonato de
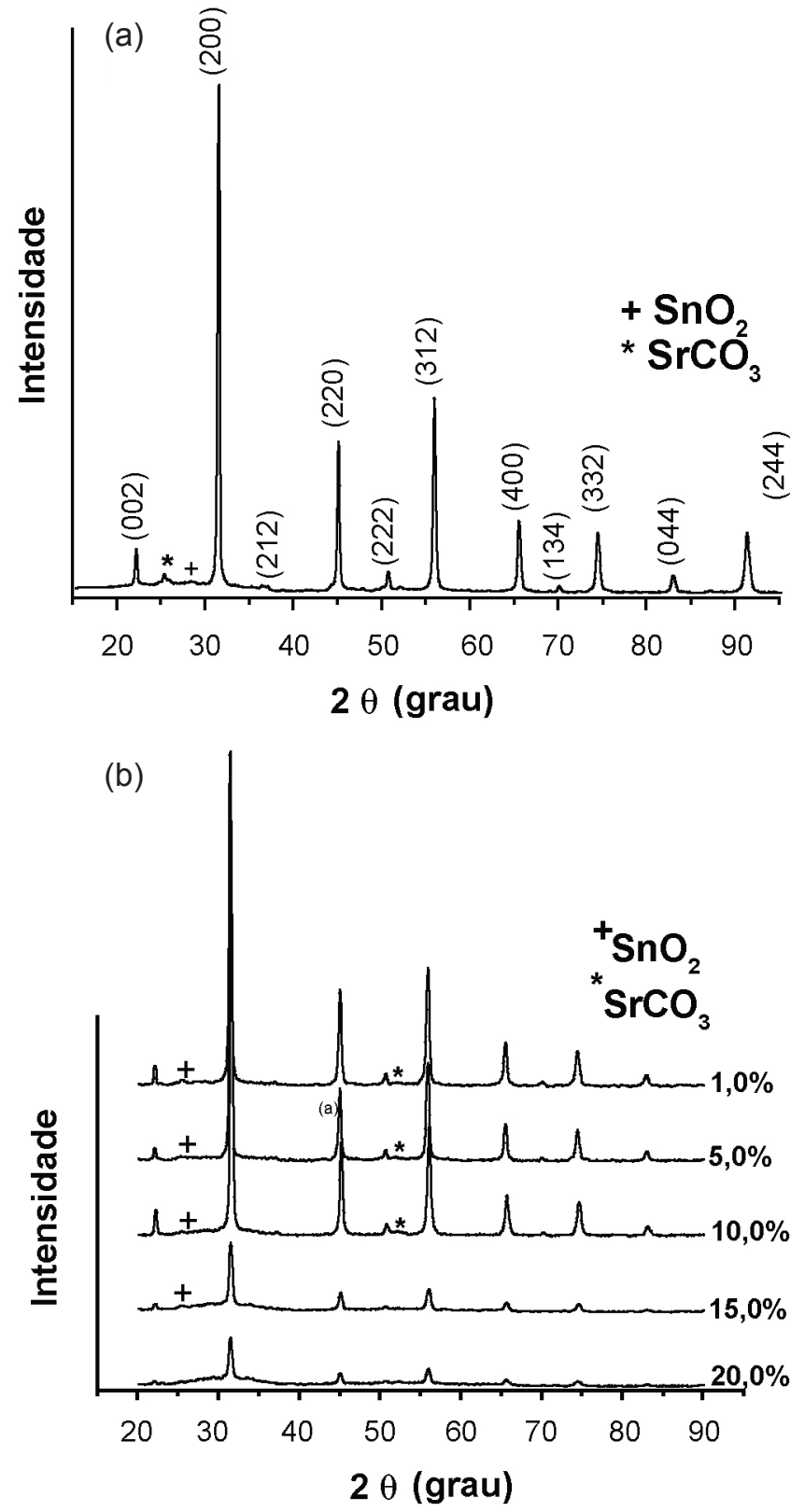

Figura 3: Difratogramas de raios $\mathrm{X}$ de (a) $\mathrm{SrSnO}_{3}$ puro calcinado a $700{ }^{\circ} \mathrm{C}$, (b) $\mathrm{Sr}_{1-\mathrm{x}} \mathrm{Ni}_{\mathrm{x}} \mathrm{SnO}_{3}(\mathrm{x}=0,0,01,0,05,0,10,0,15$ e 0,20$)$ calcinados a $700{ }^{\circ} \mathrm{C}$.

[Figure 3: XRD patterns of (a) pure $\mathrm{SrSnO}$ calcined at $700{ }^{\circ} \mathrm{C}$, (b) $\mathrm{Sr}_{1-x} \mathrm{Ni}_{x} \mathrm{SnO}_{3}(x=0,0.01,0.05,0.10,0.15$ and 0.20) powders calcined at $700^{\circ} \mathrm{C}$.]

estrôncio $\left(\mathrm{SrCO}_{3}\right)$, este último já observado nos resultados de infravermelho. A amostra contendo $20 \% \mathrm{de} \mathrm{Ni}^{2+}$ apresenta uma baixa cristalização, sendo, portanto, difícil de indicar a presença dessas fases secundárias. Quando $\mathrm{Ni}^{2+}$ substitui o modificador da rede, $\mathrm{Sr}^{2+}$, o aumento do caráter covalente do $\mathrm{Ni}^{2+}$ provoca uma deformação na estrutura cristalina. A adição do níquel provoca transformações estruturais a curto e médio alcance que podem conduzir a uma transição de fase. Esse fenômeno é comum nas perovskitas quando se substitui o modificador da rede [36].

A equação B apresenta o balanço de carga usando a 

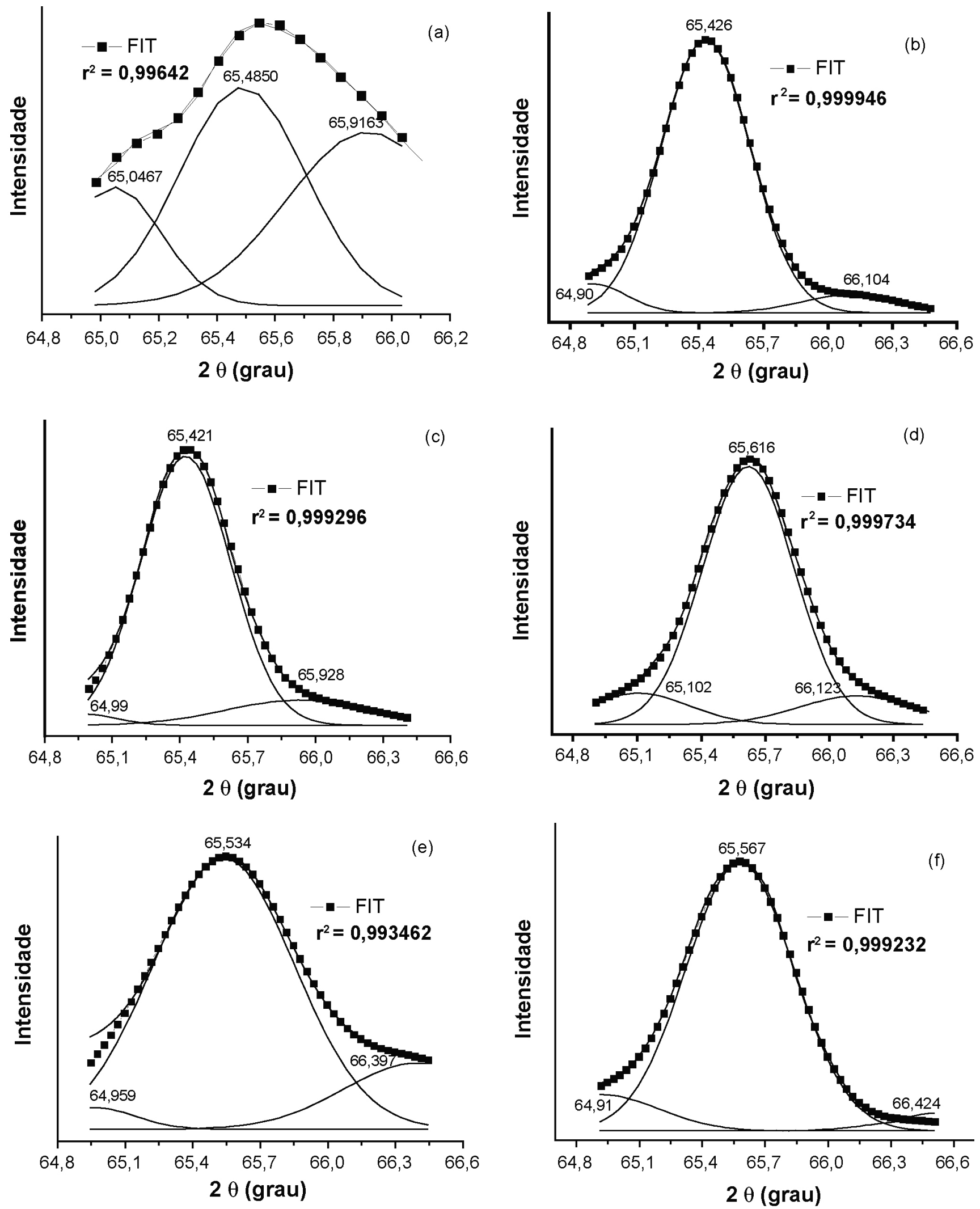

Figura 4: Deconvolução do pico $\left(\begin{array}{lll}4 & 0 & 0\end{array}\right)$ do DRX do sistema $\mathrm{Sr}_{1-\mathrm{x}} \mathrm{Ni}_{\mathrm{x}} \mathrm{SnO}_{3}$, calcinado a $700{ }^{\circ} \mathrm{C} .(\mathrm{a}) \mathrm{x}=0 ;(\mathrm{b}) \mathrm{x}=0,01 ;(\mathrm{c}) \mathrm{x}=0,05 ;(\mathrm{d}) \mathrm{x}=$ 0,10 ; (e) $\mathrm{x}=0,15$ and (f) $\mathrm{x}=0,20$.

[Figure 4: Deconvolution of the (4 00 ) peak of the XRD patterns of the $\mathrm{Sr}_{1-x} \mathrm{Ni} \mathrm{SnO}_{3}$ system, heat treated at $700^{\circ} \mathrm{C}$. (a) $x=0$; (b) $x=0.01$; (c) $x=0.05$; (d) $x=0.10$; (e) $x=0.15$ and (f) $x=0.20$.] 
notação de Kröger-Vink [37]. Eletronicamente, a impressão que se tem, a partir da análise da equação, é que nenhuma mudança ocorre. Porém, as distorções provocadas pelo aumento do caráter covalente da ligação $\mathrm{Ni}-\mathrm{O}$ (mais direcional) quando comparada a $\mathrm{Sr}-\mathrm{O}$ e as diferenças nas distâncias dessas ligações provocam mudanças nos ângulos de inclinação dos octaedros $\mathrm{SnO}_{6}$.

$$
\mathrm{NiO} \stackrel{\mathrm{SrSnO}_{3}}{\longrightarrow} \mathrm{Ni}_{\text {Sr }}^{\mathrm{x}}+\mathrm{O}_{\mathrm{O}}^{\mathrm{x}}
$$

De acordo com testes padrões de difração de raios X, foi observada uma diminuição na cristalinidade para as amostras que contêm 15 e $20 \%$ em mol de $\mathrm{Ni}^{2+}$, indicando que, apesar da precipitação do $\mathrm{SnO}_{2}$, o níquel que consegue se acomodar na célula unitária torna a cristalização do sistema mais difícil. Pode-se inferir também que os mesmos picos de difração são observados para todas as amostras analisadas. É difícil verificar diferenças mínimas e sensíveis nesses picos e, normalmente, a atribuição da estrutura cristalina do estanato de estrôncio é feita com cálculos Rietveld ou com difração de nêutrons. Dados da literatura indicam que o $\mathrm{SrSnO}_{3}$ é ortorrômbico quando calcinado a $700{ }^{\circ} \mathrm{C}$ [38-41].

A partir da deconvolução dos picos $\left(\begin{array}{lll}4 & 0 & 0\end{array}\right)$ (Fig. 4 e Tabela III), nota-se uma diferença significativa no perfil do pico deconvoluído da amostra não-dopada com relação às amostras que contêm níquel.

Tabela III - Parâmetros do pico (4 0 0 $)$ da difração de raios $\mathrm{X}$ de $\mathrm{Sr}_{1-\mathrm{x}} \mathrm{Ni}_{\mathrm{x}} \mathrm{SnO}_{3}$ calcinado a $700{ }^{\circ} \mathrm{C}$.

[Table III - Parameters of the (4 0 O patterns of $\mathrm{Sr}_{1-x} \mathrm{Ni}_{x} \mathrm{SnO}_{3}$ calcined at $700^{\circ} \mathrm{C}$.]

\begin{tabular}{cccc}
\hline \multirow{2}{*}{ Dopante (\%) } & $\begin{array}{c}\text { posição } \\
\text { do pico } \\
\text { (grau) }\end{array}$ & $\begin{array}{c}\text { Amplitude } \\
\text { normalizada }\end{array}$ & $\begin{array}{c}\text { FWHM } \\
\text { (grau) }\end{array}$ \\
\hline \multirow{2}{*}{0} & 65,05 & 0,54 & 0,38 \\
& 65,48 & 1 & 0,51 \\
& 65,92 & 0,79 & 0,66 \\
1 & 64,90 & 0,11 & 0,37 \\
& 65,43 & 1 & 0,49 \\
& 66,10 & 0,66 & 0,54 \\
5 & 64,99 & 0,040 & 0,34 \\
& 65,42 & 1 & 0,47 \\
10 & 65,92 & 0,093 & 0,76 \\
& 65,10 & 0,12 & 0,56 \\
& 65,62 & 1 & 0,50 \\
15 & 66,12 & 0,11 & 0,64 \\
& 64,96 & 0,080 & 0,42 \\
& 65,53 & 1 & 0,74 \\
\multirow{2}{*}{20} & 66,40 & 0,24 & 0,77 \\
& 64,91 & 0,12 & 0,41 \\
& 65,57 & 1 & 0,63 \\
& 66,42 & 0,040 & 0,31 \\
\hline
\end{tabular}

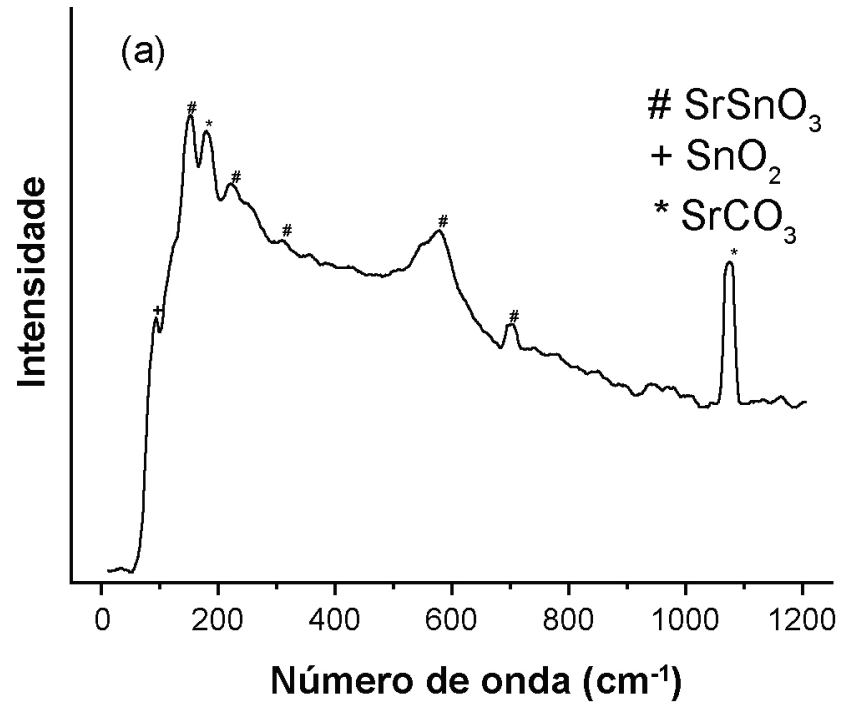

(b)

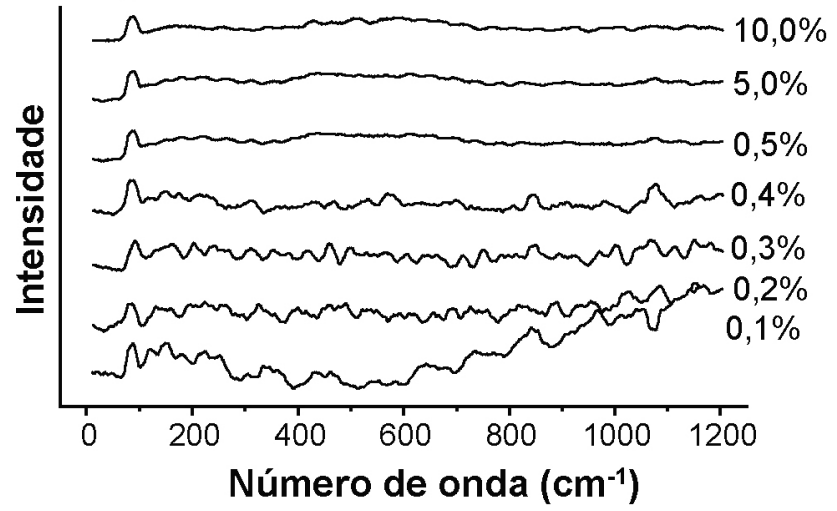

Figura 5: Espectros Raman de pós de $\mathrm{Sr}_{1-\mathrm{x}} \mathrm{Ni}_{\mathrm{x}} \mathrm{SnO}_{3}$ calcinado a $700{ }^{\circ} \mathrm{C}$ : (a) $\mathrm{x}=0$, (b) $\mathrm{x}=0,001,0,002,0,003,0,004,0,005,0,05$ e 0,10 .

[Figure 5: Raman spectra of the $\mathrm{Sr}_{1-x} \mathrm{Ni}_{x} \mathrm{SnO}_{3}$ powders heat treated at $700^{\circ} \mathrm{C}$ : (a) $x=0$, (b) $x=0.001,0.002,0.003,0.004,0.005,0.05$ and 0.10.$]$

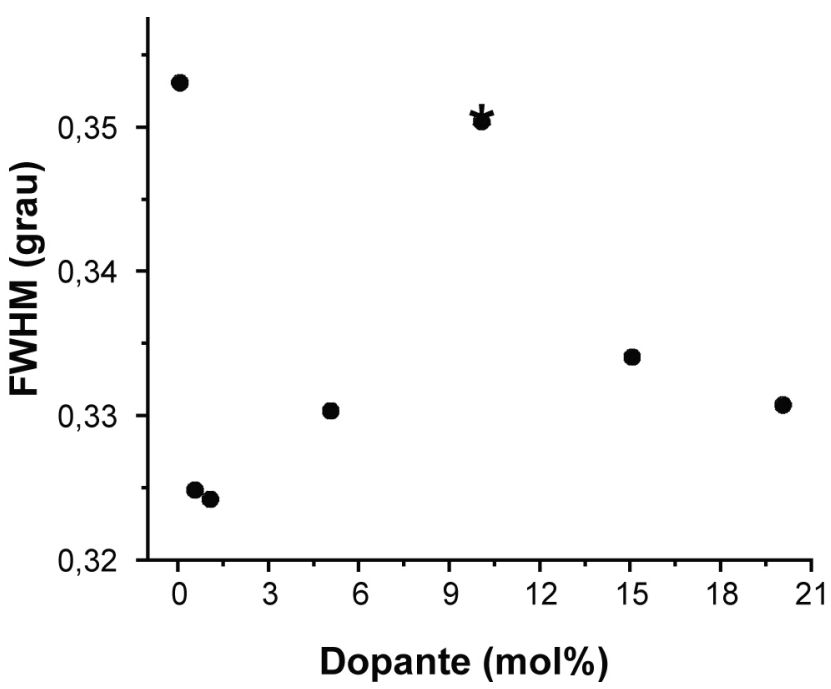

Figura 6: Valores de FWHM para $\mathrm{Sr}_{1-\mathrm{x}} \mathrm{Ni}_{\mathrm{x}} \mathrm{SnO}_{3}$ calcinado a $700{ }^{\circ} \mathrm{C}$. [Figure 6: FWHM values of the $\mathrm{Sr}_{1-x} \mathrm{Ni}_{x} \mathrm{SnO}_{3}$ powders calcined at $\left.700^{\circ} \mathrm{C}.\right]$ 
Os distintos perfis desses picos são indícios das distorções estruturais sofridas pela rede cristalina a longo alcance, a fim de buscar o equilíbrio do sistema. Para a amostra não-dopada a deconvolução produziu três picos principais, indicando

(a)

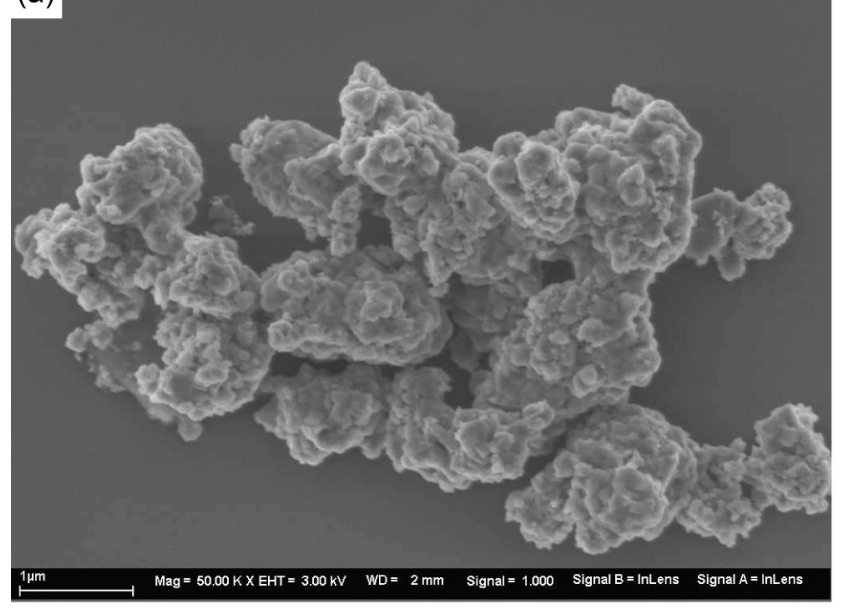

(c)

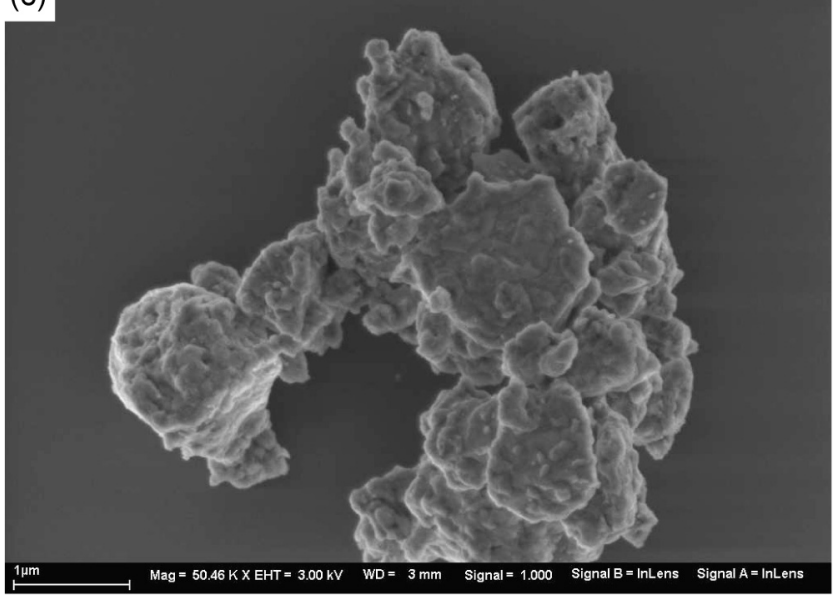

uma estrutura ortorrômbica. Diferente desse comportamento, as amostras dopadas exibiram um pico central com elevada amplitude e dois picos menores localizados em cada um dos lados do pico central. Os sinais desses picos menores
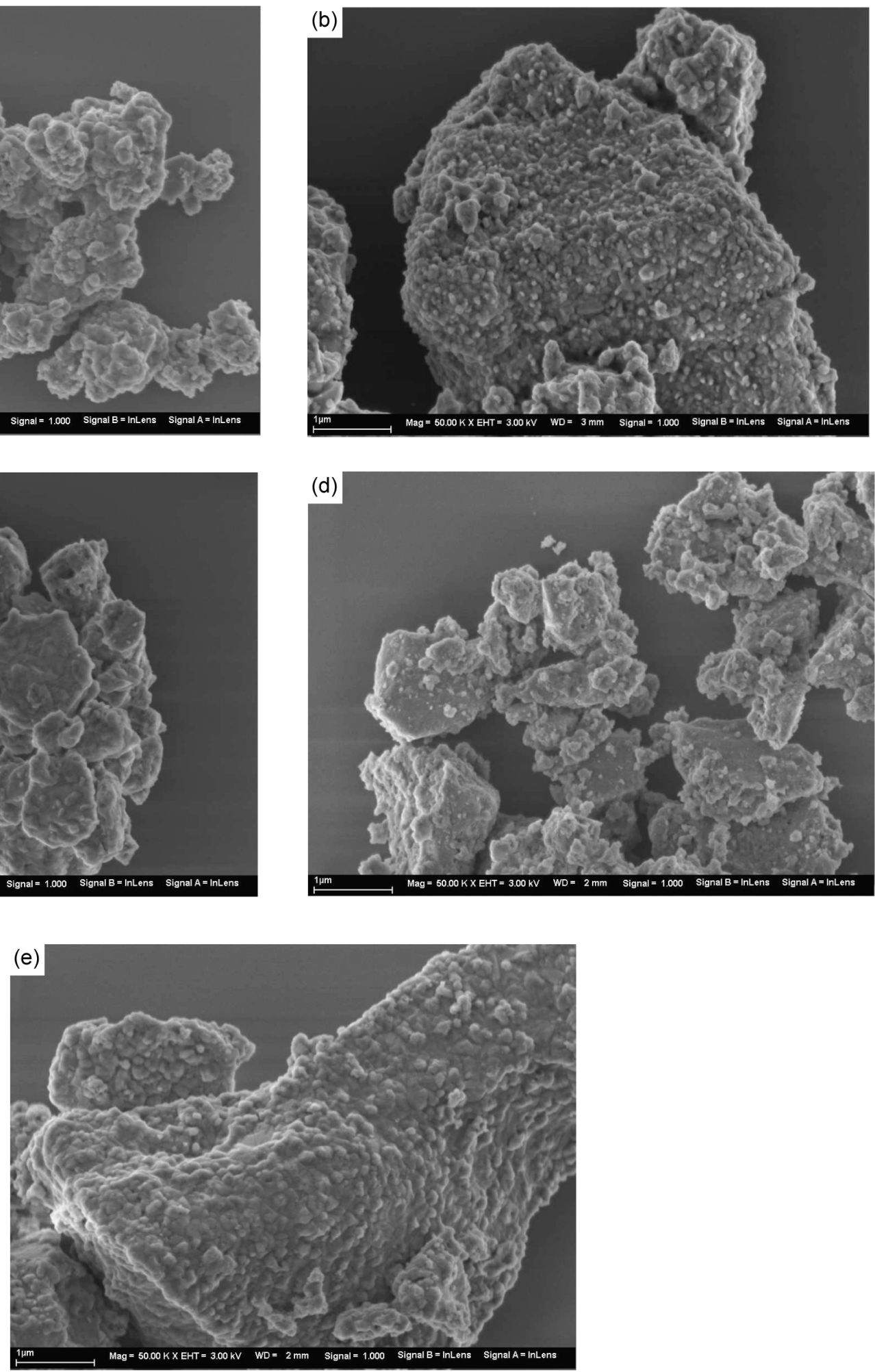

Figura 7: Micrografias MEV do sistema $\mathrm{Sr}_{1-\mathrm{x}} \mathrm{Ni}_{\mathrm{x}} \mathrm{SnO}_{3}$, calcinado a $700{ }^{\circ} \mathrm{C}$ (a) $\mathrm{x}=0,(\mathrm{~b}) \mathrm{x}=0,005$, (c) $\mathrm{x}=0,01,(\mathrm{~d}) \mathrm{x}=0,05 \mathrm{e}(\mathrm{e}) \mathrm{x}=0,10$.

[Figure 7: SEM micrographs of the $\mathrm{Sr}_{1-x} \mathrm{Ni}_{x} \mathrm{SnO}_{3}$ samples calcined at $700^{\circ} \mathrm{C}:($ a) $x=0$, (b) $x=0.005$, (c) $x=0.01$, (d) $x=0.05$ and (e) $x=0.10$.] 
se confundem com a radiação de fundo, sendo, portanto, desprezíveis. Essa avaliação mostra que o sistema se organizou a longo alcance, pois os picos de difração se tornaram mais definidos e há menos picos secundários com amplitudes consideráveis. Esse comportamento indica que uma transição de fase pode estar ocorrendo.

Os espectros Raman do $\mathrm{SrSnO}_{3}$ puro, calcinado a $700{ }^{\circ} \mathrm{C}$ (Fig. 5), apresentam bandas alargadas, que não são identificadas facilmente, devido à sobreposição de picos menos intensos. Estas bandas são observadas em 150,0, 221,5, 252,0, 308,0, 576,0 e $705 \mathrm{~cm}^{-1}[9,28,29]$. Uma banda atribuída ao $\mathrm{SnO}_{2}$ (cassiterita) pode ser identificada em $91 \mathrm{~cm}^{-1}$ (modo $\mathrm{B}_{2 \mathrm{~g}}$ ), concordando com os difratogramas de raios X. Além desta, duas bandas são atribuídas ao carbonato de estrôncio $\left(\mathrm{SrCO}_{3}\right)$, identificadas em torno de $182,4 \mathrm{~cm}^{-1}$ (forte) (modo $\mathrm{B}_{2 \mathrm{~g}}$ ) e $1071,6 \mathrm{~cm}^{-1}$ (muito forte), com modo de vibração $\mathrm{A}_{1 \mathrm{~g}}$, devido ao estiramento da ligação C-O. Outras bandas que podem ser identificadas em torno de $980 \mathrm{~cm}^{-1} \mathrm{e} 1160 \mathrm{~cm}^{-1}$ (largas e fracas) não foram indexadas e ainda não há registro na literatura sobre os modos vibracionais e os grupos responsáveis por tais vibrações. Quando níquel é adicionado ao material, nota-se que são gerados espectros Raman muito diferentes do sistema nãodopado. Com quantidades de níquel de até $0,4 \%$ é observado um aumento no ruído. Quando se insere uma maior quantidade do dopante ao sistema, a banda em $84,9 \mathrm{~cm}^{-1}$ ainda é observada, relacionada ao $\mathrm{SrSnO}_{3}$, além de algumas bandas muito largas em aproximadamente 215 e $550 \mathrm{~cm}^{-1}$.

O aparecimento de largas bandas no espectro Raman indica a presença de uma fase pseudo-cúbica $[9,28,29]$. Estas bandas alargadas indicam também que ocorre uma desordem a curto alcance. Isto ocorre provavelmente devido à presença concomitante dos grupos $\left[\mathrm{SrO}_{12}\right]$ e $\left[\mathrm{NiO}_{12}\right]$ e ao maior caráter covalente das ligações $\mathrm{Ni}^{2+}-\mathrm{O}^{2-}$.

A Fig. 6 apresenta os dados da largura à meia altura, que avalia a organização do sistema a médio e longo alcance, para o pico 100\% (2 0 0), antes e depois da dopagem. Quando o dopante é adicionado ao material é observado um aumento na periodicidade a longo alcance (o valor de FWHM diminui), com exceção da amostra com 10\% em mol de dopante. Esta periodicidade mais elevada a longo alcance é relacionada com a menor simetria a curto alcance, como indicado pelos espectros Raman e espectros no infravermelho.

As microestruturas das amostras dopadas e não-dopadas são apresentadas na Fig. 7. Os resultados indicam que há uma larga distribuição no tamanho da partícula, com a ocorrência de grandes agregados. Pode-se observar que as partículas submicrométricas formam agregados de até $200 \mu \mathrm{m}$. A adição do dopante não muda a formação dos agregados nem o tamanho das partículas. O processo de sinterização ocorre de forma similar para todas as amostras calcinadas a $700{ }^{\circ} \mathrm{C}$.

\section{CONCLUSÕES}

Um método de síntese que utiliza baixas temperaturas (método dos precursores poliméricos) foi aplicado com sucesso para a síntese do $\mathrm{SrSnO}_{3}$ dopado com níquel. Para o $\mathrm{SrSnO}_{3}$ puro, a perovskita ortorrômbica foi obtida a $700{ }^{\circ} \mathrm{C}$. Quando níquel é adicionado é verificada uma mudança no perfil dos difratogramas. O aumento na porcentagem de dopante aumenta a desordem a curto alcance e diminui a desordem a longo alcance, provocando a saída parcial do estanho da estrutura perovskita, com a precipitação conseqüente da fase $\mathrm{SnO}_{2}$ (cassiterita).

\section{AGRADECIMENTOS}

À CAPES e ao CNPq/MCT pelo suporte financeiro.

\section{REFERÊNCIAS}

[1] V. Jayaraman, G. Mangamma, T. Gnanasekaran, G. Periaswami, Solid State Ionics 86-88 (1996) 1111-1 114.

[2] M. Licheron, G. Jouarf, E. Hussona, J. Eur. Ceram. Soc. 17 (1997) 1453-1457.

[3] S. V. Manorama, C. V. Gopal Reddy, V. J. Rao, Appl. Surf. Sci. 174 (2001) 93-105.

[4] J. Cerdà, J. Arbiol, G. Dezanneau, R. Díaz, J. R. Morante, Sensor Actuat. B-Chem 84 (2002) 21-25.

[5] U. Lampe, J. Gerblinger, H. Meixner, Sensor Actuat. BChem. 25 (1995) 657-660.

[6] Om. Parkash, D. Kumar, K. K. Srivastav, R. K. Dwivedi, J. Mater. Sci.: Mater. Electr. 36 (2001) 5805-5810.

[7] M. D. Águas, L. Morris, I. P. Parkin, J. Mater. Sci. 37 (2002) 375-379.

[8] C. V. G. Reddy, S. V. Manorama, V. J. Rao, J. Mater. Sci. 12 (2001) 137-142.

[9] J. Cerdà, J. Arbiol, R. Diaz, G. Dezanneau, J. R. Morante, Mater. Lett. 56 (2002) 131-136.

[10] A.-M. Azad, J. Mater. Sci. 36 (2001) 3909-3917.

[11] A. A. Al-Shahrani, J. Mater. Sci.: Mater. Electr. 16 (2005) 193-196.

[12] A.-M. Azad, M. Hashim, S. Baptist, A. Badri, A. Ul. Haq, J. Mater. Sci. 35 (2000) 5475-5483.

[13] A-M. Azad, L. L. W. Shyan, T. Y. Pang, C. H. Nee, Ceram. Int. 26 (2000) 685-692.

[14] A.-M. Azad, L.J. Min, M. A. Alim, Ceram. Int. 27 (2001) 335-341.

[15] S. Hodjati, K. Vaezzadeh, C. Petit, V. Pitchon, A. Kiennemann, Appl. Catal B- Environ. 26 (2000) 5-16.

[16] T. Ishihara, H. Fujita, H. Nishiguchi, Y. Takita, Sensor Actuat B-Chem. 65 (2000) 319-324.

[17] T. Ishihara, H. Fujita, Y. Takita, Sensor Actuat B-Chem. 52 (1998) 100-106.

[18] Q. Jing, H. Lou, J. Fei, Z. Hou, X. Zheng, Int. J. Hydrogen En. 29 (2004) 1245-1251.

[19] J. Kirchnerova, D. Klvana, Solid State Ionics 123 (1999) 307-317.

[20] G. Valderrama, M. R. Goldwasser, C. U. de Navarro, J. M. Tatibouet, J. L. Barrault, C. B.-Dupeyrat, F. Martínez, Catal. Today 107-108 (2005) 785-791.

[21] F.-C. Buciuman, F. Patcas, J.-C. Menezo, J. Barbier, T. Hahn, H.-G. Lintz, Appl. Catal B-Environ. 35 (2001) 149-156. [22] Y. Cesteros, R. Fernandez, J. Estellé, E. Salagre, F. Medina, J. E. Sueiras, J. L. G. Fierro, Appl. Catal A-Gen. 152 (1997) 249-269. 
[23] A.-M. Azad, L. L. W. Shyan, P.T. Yen, J.Alloys Compd. 282 (1999) 109-124.

[24] S. Wang, M. Lu, G. Zhou, Y. Zhou, A. Zhang, Z. Yang, J.Alloy Compd. 432 (2007) 265-268.

[25] Z. Lu, L. Chen, Y. Tang, Y. Li, J. Alloys Compd. 387 (2005) L1-L4.

[26] G. Pfaff, Thermochimica Acta 237 (1994) 83-90.

[27] M. P. Pechini, United States Patent n. 3.330.697 (July 1967).

[28] M. Kakihana, M. Yoshimura, Bull. Chem. Soc. Jpn. 72 (1999) 1427-1443.

[29] C. P. Udawatte, M. Kakihana, M. Yoshimura. Solid State Ionics 128 (2000) 217-226.

[30] S. M. Zaneti, E. Longo, J. A. Varela, E. R. Leite, Mater. Lett. 31 (1997) 173-178.

[31] C. P. Udawatte, M. Kakihana, M. Yoshimura, Solid State Ionics 108 (1998) 23-30.

[32] C. O. Paiva Santos, D. Garcia, Y. P. Mascarenhas, J. A. Eiras, Cerâmica 35 (1989) 153-157.

[33] D. S. Gouveia, R. Rosenhaim, M. A. M. A. Maurera, S. J. G. Lima, C. A. Paskocimas, E. Longo, A. G. Souza, I. M.
G. Santos, J. Thermal Analysis and Calorimetry 75 (2004) 453-460.

[34] M. Licheron, G. Jouarf, E. Hussona, J. Eur. Ceram. Soc. 17 (1997) 1453-1457.

[35] R. Nyquist, R. Kagel, Infrared Spectra of Inorganic Compounds (3800-45 cm $\mathrm{cm}^{-1}$ ), Academic Press Inc., N. York (1971).

[36] E. H. Mountstevens, S. A. T. Redfern, J. P. Attfield, J. Phys.: Cond. Matter 15 (2003) 8315-8326.

[37] Y. M. Chiang, D. P. Birnie III, W. D. Kingery, Physical Ceramics - Principles for Ceramic Science and Engineering, John Wiley \& Sons Inc., N. York (1997) pp. 110-111.

[38] M. A. Green, K. Prassides, P. Day, D.A. Neumann, Int. J. Inorg. Mater. 2 (2000) 35-41.

[39] E. H. Mountstevens, S. A. T. Redfern, J. P. Attfield, Phys. Rev. B 71 (2005) 220102.

[40] M. Glerup, K. S. Knight, F. W. Poulsen, Mater. Res. Bull. 40 (2005) 507-520.

[41] H. Mizoguchi, H. W. Eng, P. M. Woodward, Inorg. Chem. 43 (2004) 1667-1680.

(Rec. 18/06/2007, Ac. 31/08/07) 